\title{
Liver reconstruction on the chorioallantoic membrane of the chick embryo
}

\author{
Akina Chiba, Chisato Yui and Shigeki Hirano \\ Department of Medical Technology, Graduate School of Health Sciences, Niigata University, Niigata, Japan
}

\begin{abstract}
Summary. The liver from a 6-day-old chick embryo was transplanted on the chorioallantoic membrane of a 9-day-old chick embryo to observe the process of liver regeneration histologically. When a piece of the liver was implanted on the chorioallantoic membrane, only cells in the superficial zone of the graft adhering to the chorioallantoic membrane survived. Eventually, these surviving cells in the superficial zone proliferated with hematopoiesis, resulting in the formation of clusters of blood cells surrounded by the hepatocytes (or hepatic parenchymal cells). Semi-thin serial sections showed that these clusters of blood cells were confined to the space formed by hepatocytes. Furthermore, structures similar to the hepatic cord, sinusoid, central vein, and bile duct appeared in the reconstructed liver eleven days after transplantation. Meanwhile, when a pellet of the dissociated liver cells was transplanted onto the chorioallantoic membrane, two types of liver like structures were reconstructed: one was clusters of hepatocytes accompanied by sinusoids after hematopoiesis, and the other was a simple accumulation of hepatocytes without any sinusoids or hematopoiesis. The sinusoids found in the former type became clear following the connection between the space in the transplant and vessels of the chorioallantoic membrane. These findings indicated that the reconstructed liver was primarily produced by the accumulation of hepatocytes accompanied by hematopoietic cells, followed by the formation
\end{abstract}

Received May 26, 2009; revised January 1, 2010

Address for correspondence: Shigeki Hirano, Ph.D. Department of Medical Technology, Graduate School of Health Sciences, Niigata University, 746, Asahimatidori-2, Chuo-ku, Niigata 951-8518, Japan

Tel: +81-25-227-0961, Fax: +81-25-227-0961

E-mail: hiranosg@clg.niigata-u.ac.jp of sinusoidal spaces. We therefore consider that hematopoiesis is important for liver regeneration with a normal structure. Transplantation of the liver in the chorioallantoic membrane could be also useful for research into liver regeneration.

\section{Introduction}

The liver has an extensive ability to regenerate: even if two-thirds of the liver is eliminated, it will be restored to its full size within a relatively short period of time (Michalopoulos and DeFrances, 1997). This ability is most apparent abdomen, which is thought to provide the optimum environment for liver regeneration. Thus, many studies have been performed over years to examine the reconstruction of the liver in vivo as well as in vitro. For example, hepatic tissues have been transplanted into the intraperitoneal space (Shibata et al., 2006), spleen (Mito et al., 1979), subcutaneous (Ohashi et al., 2007), and other tissues. Dissociated hepatic cells have also been cultured in vitro on artificial membranes and other scaffolding materials (Harada et al., 2003; Sudo et al., 2005; Wen et al., 2008; Feng et al., 2009 ). The results of these studies indicated that the process of liver regeneration after partial hepatectomy differs from the in vivo and/or in vitro transplantation experiments.

The chorioallantoic membrane of the chick has often been used experimentally as a host for an organ culture (Hancox, 1947; Sezer, 1950; Nijweide et al., 1982; Bertossi et al., 1998; Nakada et al., 1998). This membrane consists of an outer ectodermal layer and an inner endodermal layer, with a mesodermal layer between. Numerous blood vessels develop in the mesodermal layer and are believed to supply nourishment to the transplanted tissue. Because the chorioallantoic membrane is located directly under the eggshell, it is also very useful for transplantation by making a 
window on the eggshell to observe the state of the transplanted materials at any given time. As compared with transplantation in a culture dish where only one type of cells can survive over a long period, transplantation on the chorioallantoic membrane is highly beneficial for regeneration studies because many different cell types can develop simultaneously on the membrane. Moreover, there is no rejection of the transplantation because the immune system is not yet functional in 9-day-old chick embryos. Thus, Murphy (1916) used the chorioallantoic membrane as a host organ for tissue transplantation and investigated various chicken tissues grafted on this membrane. Katoh et al. (2001) also transplanted liver pieces of the chick embryos onto the chorioallantoic membrane and showed that the liver was able to regenerate on this membrane. However, no histological details for the process of liver regeneration were described in their article.

The liver is formed by a cluster of lobules that act as a functional unit. The major component of hepatic lobules is hepatocytes (i.e., hepatic parenchymal cells), but there are also other types of cell, such as Ito cells, Kupper cells, sinusoidal endothelial cells, and biliary epithelial cells. To accomplish complete hepatic function, development of the normal structure of hepatic lobules is required. However, obtaining a regenerated liver having both a normal structure and suitable size for transplantation would have great significance for future clinical treatments requiring liver transplantation.

Thus, the aim of the present study was to investigate in detail the process of liver regeneration after transplantation of an embryonic chick liver grafted onto the chorioallantoic membrane. Based on the results obtained in this study, we propose that hematopoiesis plays an important role in liver regeneration.

\section{Materials and Methods}

\section{Preparation of host eggs}

Fertilized white leghorn chicken eggs were laid and incubated at $37.5^{\circ} \mathrm{C}$ in $58 \%$ humidity in an egg incubator. After incubation for $48 \mathrm{~h}$, the albumin was removed from the egg. A round window was opened in the eggshell and sealed using cellophane tape. The eggs were then incubated until $\mathrm{H}-\mathrm{H}$ stage 35 (about nine days) (Hamburger and Hamilton, 1951). Prior to transplantation, the tape on the window was detached and the epithelial layer of the chorioallantoic membrane was removed as follows. First, a $5 \mathrm{~mm}$ square piece of filter paper that had been moistened with distilled water was frosted using

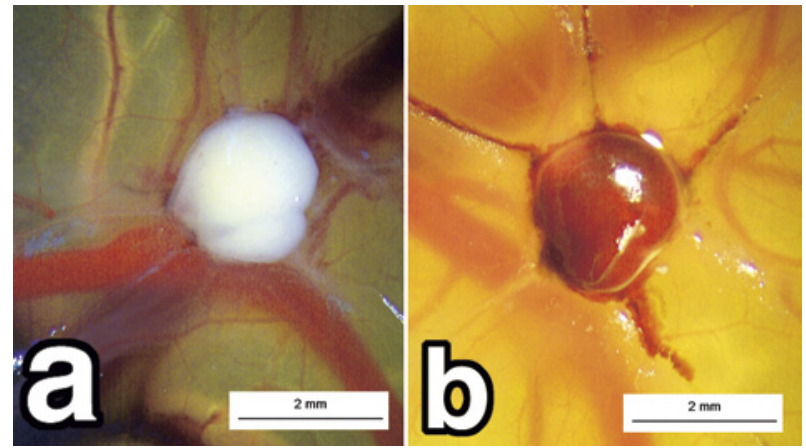

Fig. 1. Overview of the liver implant after transplantation. a: The graft just after transplantation is whitish. b: The grafted liver changes to red at two days post-transplantation, suggesting the presence of hematopoiesis.

liquid nitrogen. Then the filter paper was pressed onto the chorioallantoic membrane. After defrosting, the epithelial layer of the chorioallantoic membrane was removed using a tungsten needle, thereby exposing the mesodermal layer of the membrane.

\section{Preparation of donor eggs and transplantation}

Donor eggs were incubated using the same conditions as the host until H-H 29 (about six days), then the developing whole liver was taken and grafted onto the mesoderm of the chorioallantoic membrane in the host egg (H-H stage 35). For the transplantation of dissociated liver cells, in contrast, whole liver cells derived from the H-H 29 embryo were dissociated by passing them through a sheet mesh attached to the tip of a handcrafted tube with a $1 \mathrm{~m} l$ Terumo syringe (TERUMO, Tokyo). A droplet of the liver cells was dropped and spread on the chorioallantoic membrane. To prevent the loss of the grafted cells by their flowing out, the grafted region was covered with a $9 \mathrm{~mm}^{2}$ glass plate. After transplantation, $0.1 \mathrm{~m} l$ of saturated erythromycin was dropped into each egg, the round window was once again sealed with cellophane tape, and the egg was placed back in the incubator.

\section{Histological observations}

The grafted liver pieces with the neighboring chorioallantoic membrane were excised from the eggs 1 to 11 days after transplantation, and fixed with $10 \%$ formaldehyde in a $0.01 \mathrm{M}$ phosphate buffered saline (PBS) $(\mathrm{pH} 7.3)$ for $24 \mathrm{~h}$ at room temperature. The specimens were dehydrated in an ethanol gradient from $70 \%$ to absolute, cleared in xylene, and embedded in paraffin. 
Half of the blocks were then sliced into $12 \mu \mathrm{m}$ serial sections, which were stained with hematoxylin-eosin for histological examination. The remaining samples were sliced into $3 \mu \mathrm{m}$ sections for immunohistochemical examination. In order to investigate the connections between blood vessels in the chorioallantoic membrane and the graft, India ink was injected into the vessels of the chorioallantoic membrane.

TUNEL analysis was performed to assess cell death. The grafts were fixed with $10 \%$ formaldehyde in $0.01 \mathrm{M}$ PBS, embedded in paraffin, and sliced into $3 \mu \mathrm{m}$ sections. The deparaffinized sections were rehydrated through an ethanol series and incubated with $3 \% \mathrm{H}_{2} \mathrm{O}_{2}$ for 15 min, $0.1 \%$ NP40 for $15 \mathrm{~min}$, and a TUNEL medium $(0.16 \%$ cacodylate sodium, $0.129 \% \mathrm{CoCl}_{2}, 0.05 \%$ gelatin, 10 $\mathrm{nmol} / \mathrm{m} l$ biotin-16-dUTP (Roche Diagnostics, Germany), $100 \mathrm{U} / \mathrm{m} l$ terminal deoxynucleotidyl transferase pH7.0 (TOYOBO, Osaka)) for $60 \mathrm{~min}$ at $37^{\circ} \mathrm{C}$. After washing with $\mathrm{PBS}$, the sections were processed using a VECTASTAIN ABC KIT (Vector Laboratories, Inc. USA). Then the sections were counterstained with hematoxylin and observed with a light microscope.

Cell proliferation and albumin production were examined with a PCNA monoclonal antibody (CHEMICON INTERNATIONAL) and $0.05 \%$ antichicken albumin (Inter-Cell technologies Inc.), respectively. Analysis of albumin production was used to investigate the function of hepatocytes. Fixation and other processes were the same as for the TUNEL analysis.

\section{Semi-thin sections}

The grafted liver pieces were fixed at 5 days after transplantation with $2.5 \%$ glutaraldehyde in $0.01 \mathrm{M}$ PBS for $1 \mathrm{~h}$ at $4^{\circ} \mathrm{C}$, followed by washing with PBS. The specimens were cut into about $1 \mathrm{~mm}^{3}$ pieces and postfixed with $1 \%$ osmium tetroxide in $0.01 \mathrm{M}$ PBS for $1 \mathrm{~h}$ at $4{ }^{\circ} \mathrm{C}$, dehydrated in a graded series $(70-100 \%)$ of ethanol, transferred to propylene oxide, and embedded in Epon-812. Semi-thin sections $(1 \mu \mathrm{m})$ were stained with
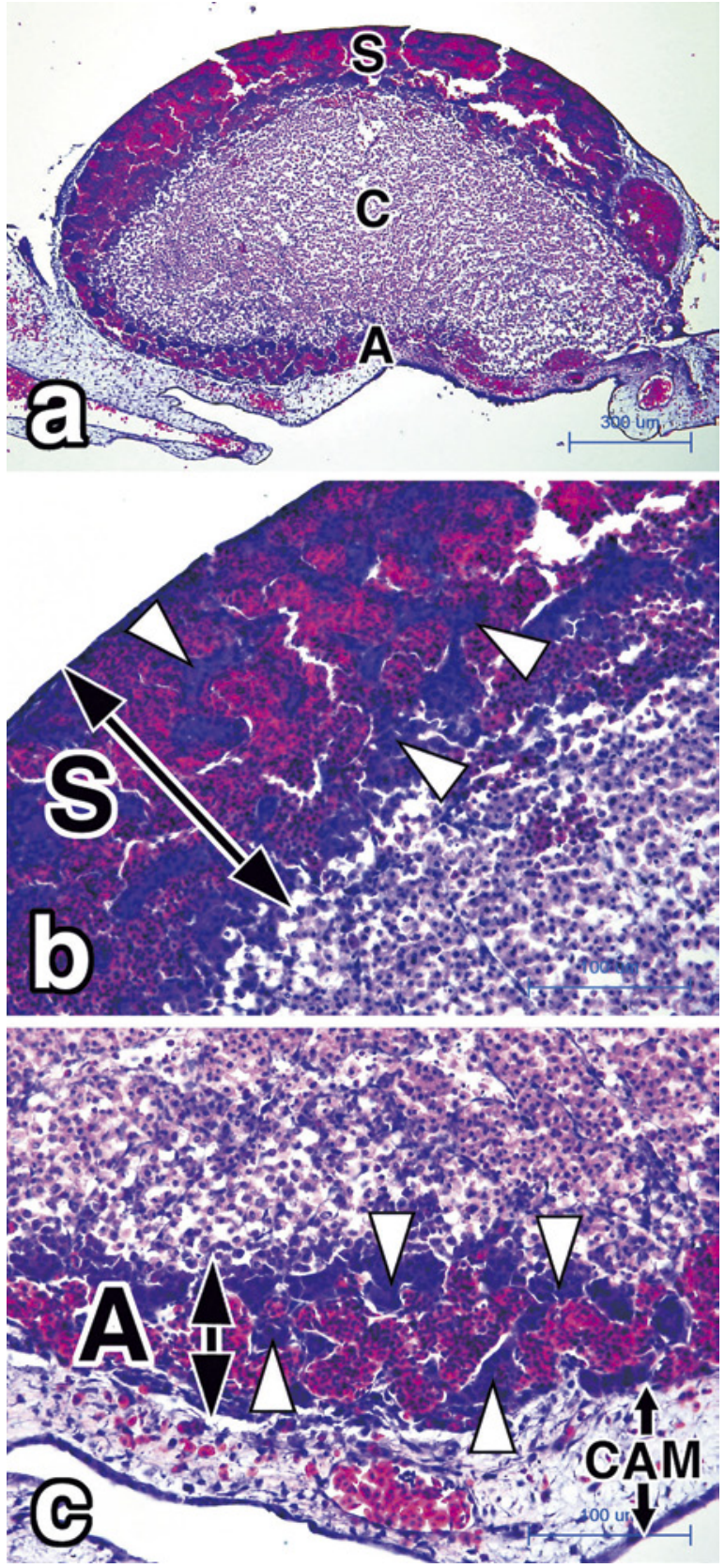

Fig. 2. Histological observations of the liver implant at one day of post-transplantation. a: The affinity of hematoxylin has decreased in the central part of the graft $(\mathrm{C})$. Both the zone of the transplant exposed to the air (S) and the zone of the transplant adhering to the chorioallantoic membrane (A) retain a high affinity for hematoxylin. The latter (A) is observed to be thinner than the former $(\mathrm{S})$. b: Closer view of the superficial zone of the transplant exposed to the air (S two-headed arrow). Hepatocytes (white arrowheads) retain an affinity for hematoxylin. In addition, many blood cells pervade among the hepatocytes. c: Closer view of the superficial zone of the transplant adhering to the chorioallantoic membrane (A two-headed arrow). The hepatocytes (white arrowheads) retain an affinity for hematoxylin, and many blood cells are found among hepatocytes. CAM indicates the chorioallantoic membrane. H-E staining 


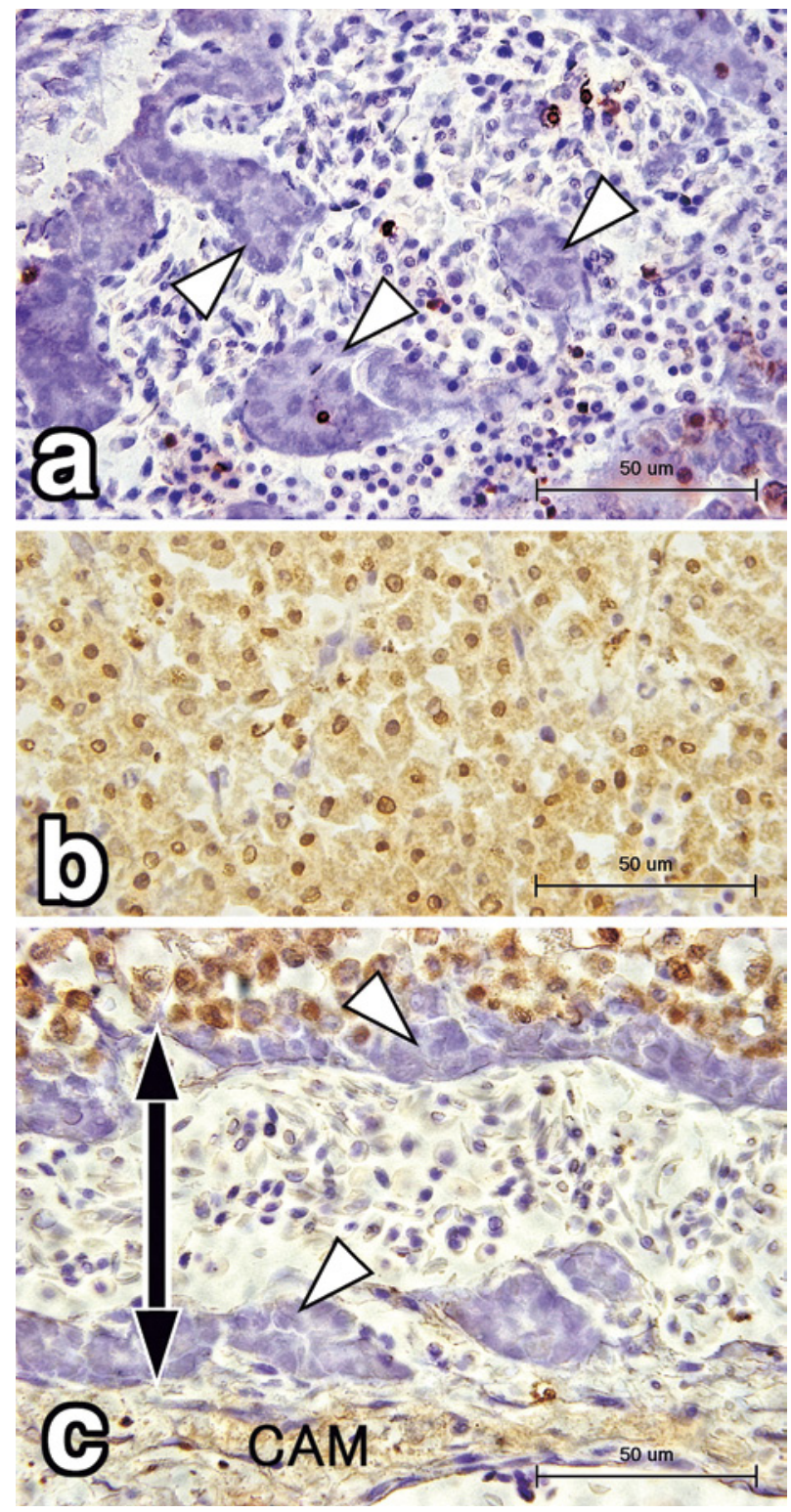

Fig. 3. TUNEL staining in the transplant at one day posttransplantation. a: The cells in the superficial zone of the transplant exposed to the air are TUNEL negative (arrowheads). b: Closer view of the central part of the graft. All cells in the central part of the graft appear TUNEL positive, indicating that the majority of the cells in the transplant die soon after transplantation. c: The cells in the superficial zone (two-headed arrow) of the transplant adhering to the chorioallantoic membrane are TUNEL negative (arrowheads). CAM indicates the chorioallantoic membrane. toluidine blue and observed with a light microscope.

\section{Results}

\section{Overview of the grafted liver after transplantation}

The whole liver of the 6-day-old chick embryo appeared whitish just after transplantation (Fig. 1a). Some of the grafts that succeeded in engrafting onto the chorioallantoic membrane turned red within a few days post-transplantation (Fig. 1b), suggesting that the graft had begun hematopoiesis. For those transplants that did not change to red within three days after transplantation, the liver pieces were not able to survive on the chorioallantoic membrane. Four days after grafting, some of the implants had been immersed into the chorioallantoic membrane.

In the transplantation of dissociated cells of the whole liver, the pellet which was dropped on the chorioallantoic membrane was also whitish just after transplantation with

Fig. 4. Histological observations of the regenerated liver after transplantation. a: Magnified figure shows the peripheral area of the reconstructed liver in the insert figure at five days after transplantation. Note numerous blood cells among the hepatocytes. Insert figure is a low magnified whole image showing the reconstructed liver. b: The central area of the reconstructed liver in the insert of figure a. There are sinusoids with wide spaces in the center of the proliferating area (asterisk). Clusters of hepatocytes (arrows) are scattered in mesenchymal tissue. Toward the central area of the reconstructed liver, the number of blood cells decreases in sinusoids (compare figures $\mathbf{a}$ and $\mathbf{b}$ ). c: Newly formed sinusoids connect to vessels of the chorioallantoic membrane (arrow). d: A reconstructed liver of a 6-day-old chick embryo at eleven days post-transplantation. The transplant shows developing structures of the liver. Note structures similar to the central vein (arrow), hepatic cord, sinusoid, and bile duct (white arrowhead). e: PCNA reactions on the region of regeneration at seven days post-transplantation. Almost all hepatocytes are positive for PCNA. f: Albumin production by hepatocytes in the regenerated liver at eleven days post-transplantation. Albumin exists for the cytoplasm of hepatocytes. g: India ink injected into a vessel in the chorioallantoic membrane appears in the sinusoidal spaces of the regenerating liver at six days post-transplantation. This result shows the anastomosis between vessels of the chorioallantoic membrane and sinusoids in the reconstructing liver. a, b, c, d, g : H-E staining 

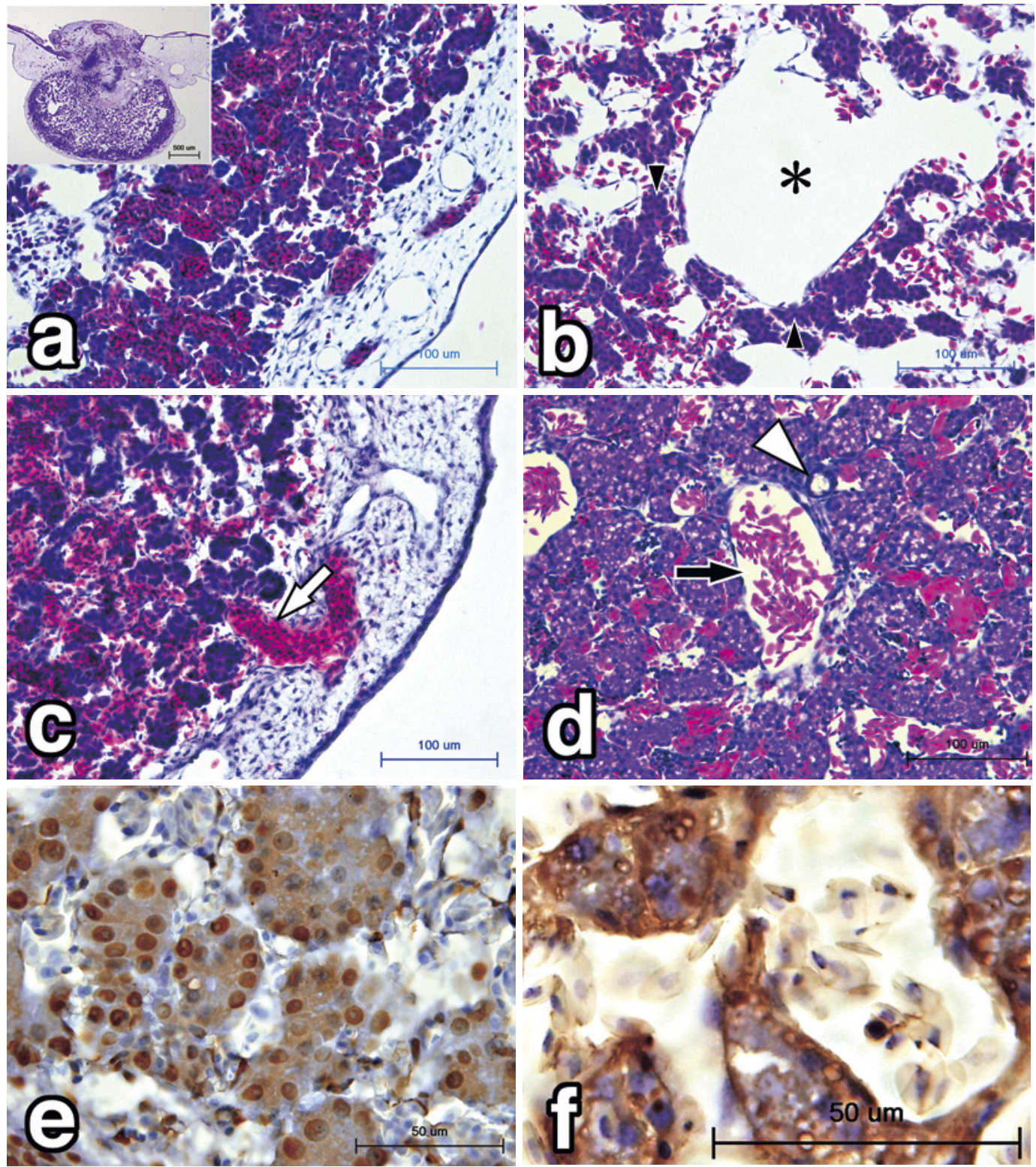

Fig. 4. Legend on the opposite page.
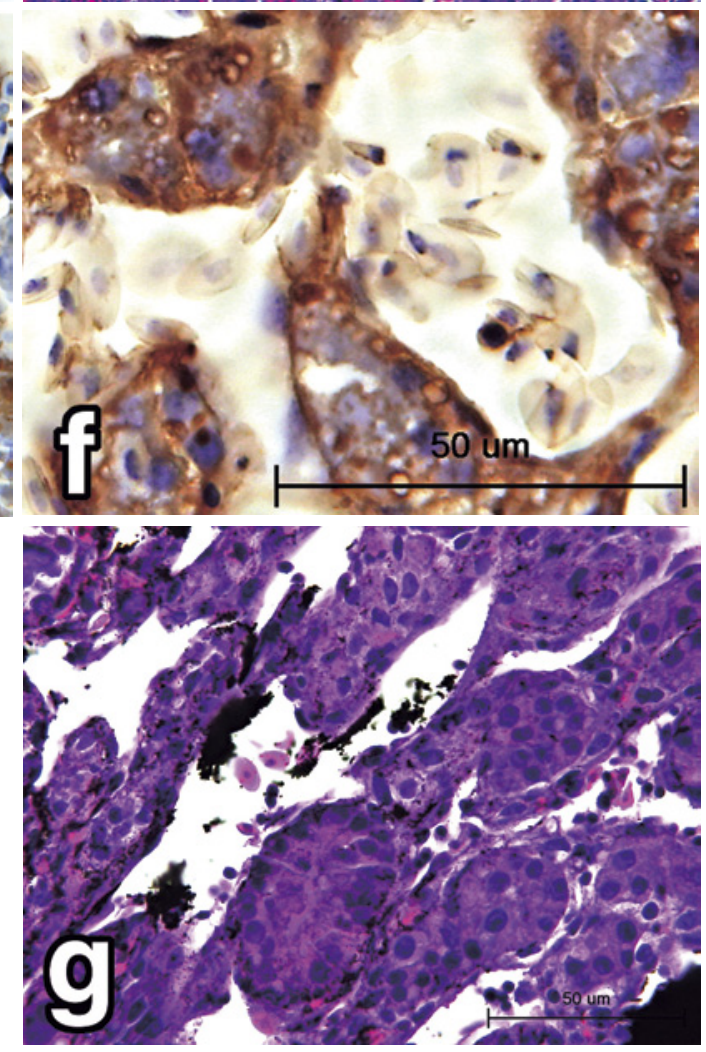

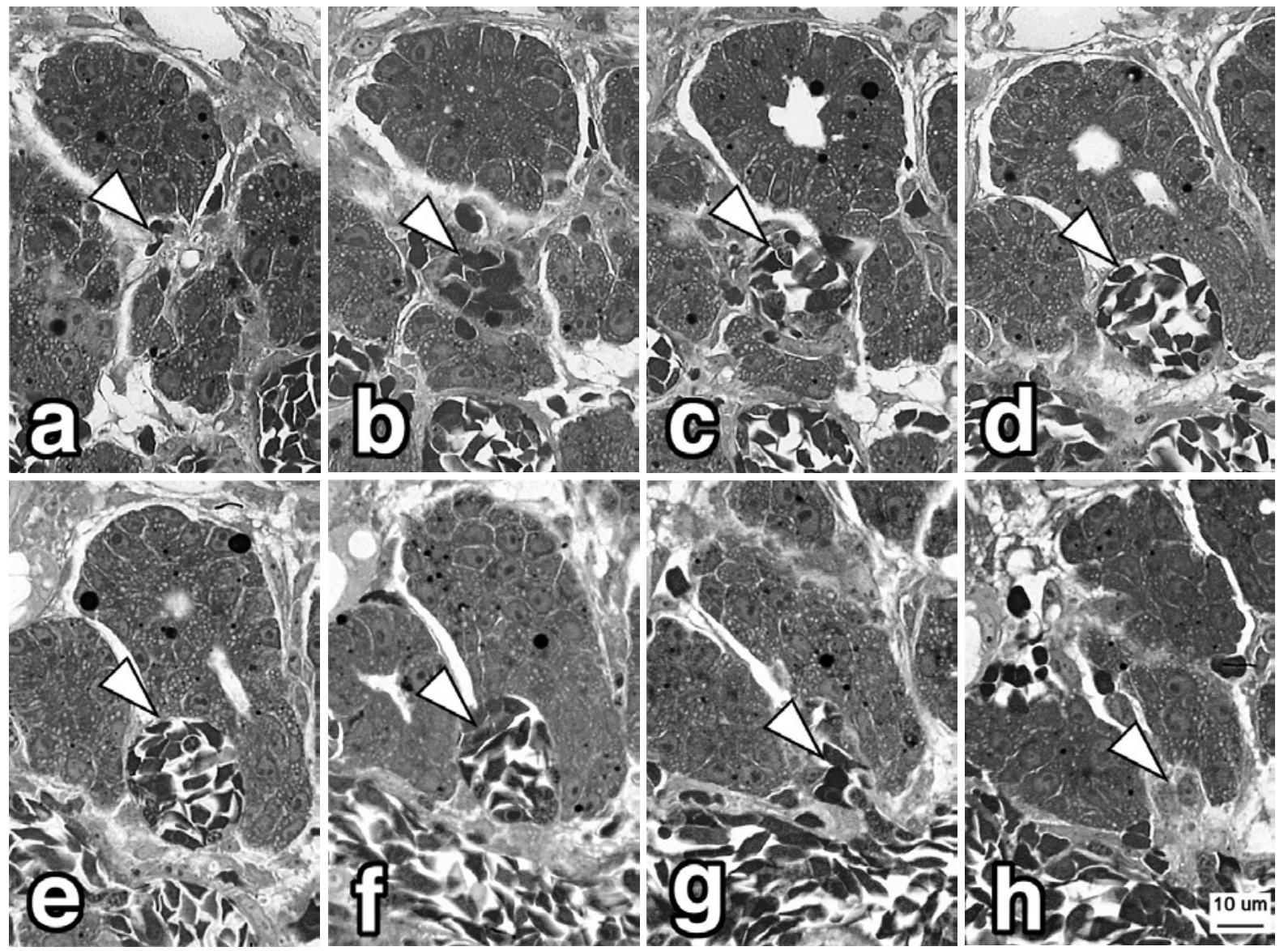

Fig. 5. Photomicrographs of serial semi-thin sections of the liver implant five days after transplantation. The serial sections were sliced at $1 \mu \mathrm{m}$ and arranged alternately to demonstrate the confinement of blood cells in a space (arrowheads). These micrographs show that hematopoiesis occurs in the reconstructing liver.

some red spots later appearing in the pellet, indicating the onset of hematopoiesis.

\section{Histological observation of grafts after transplantation}

The grafted cells derived from the 6-day-old chick embryo showed a great affinity for hematoxylin, and their cytoplasm was strongly stained violet with hematoxylineosin staining. One day after transplantation, however, this affinity for hematoxylin decreased in the large central part of the graft (Fig. 2a). TUNEL analysis revealed that most of the cells in this part were positively stained (Fig. $3 \mathrm{~b}$ ), suggesting that they were in the process of cell death. In contrast, cells in the superficial zone of the graft were TUNEL-negative (Fig. 3a, c arrowheads) and consisted of cells showing a high affinity for hematoxylin (Fig. 2b, c; arrowheads) and numerous blood cells. However, the superficial zone exposed to the air was thicker than that adhering to the chorioallantoic membrane (Fig. 2a).

By five days post-transplantation, the superficial zone exposed to the air and the central part of the graft disappeared completely. On the other hand, proliferating hepatocytes in the zone adhering to the chorioallantoic membrane occupied the entire graft (Fig. 4e), and hematopoiesis appeared throughout the proliferating area. Thereafter, active hematopoiesis was localized to the peripheral proliferating area (Fig. 4a), and sinusoids with wide spaces appeared in the center of this area (Fig. 4b; asterisk). These newly formed sinusoids in the reconstructing liver then directly connected to blood vessels in the chorioallantoic membrane (Fig. 4c arrow). An injection of India ink into a vessel in the 


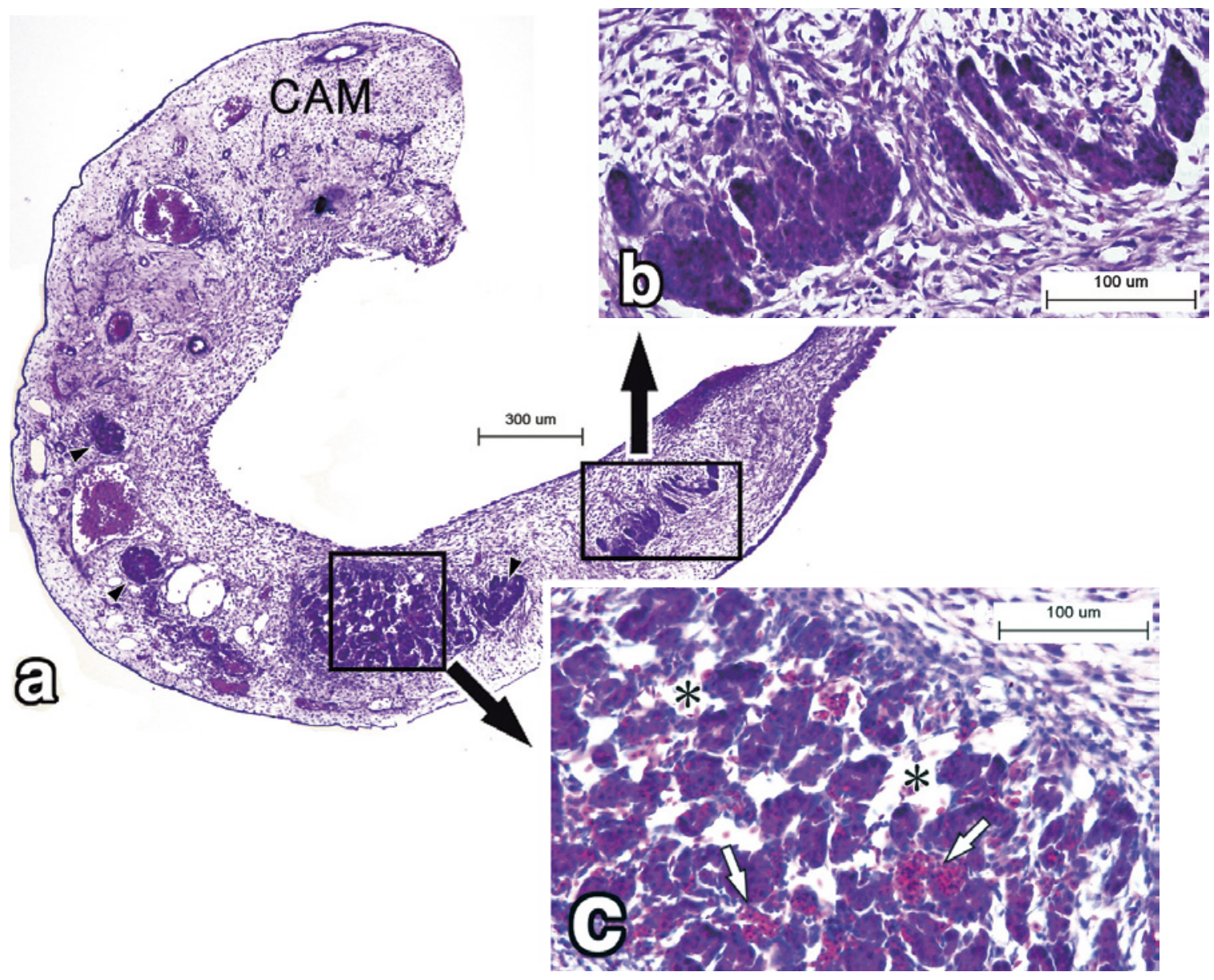

Fig. 6. Reconstructed liver derived from a pellet of the dissociated liver cells. a: Lower magnification. Arrowheads indicate a cluster of proliferated hepatocytes which are distributed in the chorioallantoic membrane (CAM). b: Magnified view enclosed by the square in $\mathbf{a}$. This picture indicates a cellular mass formed by proliferating hepatocytes alone. No sinusoid appears in the mass. c: Magnified view enclosed by the square in $\mathbf{a}$. This proliferating area is accompanied by hematopoiesis (arrows) and forming sinusoids (asterisks). H-E staining

chorioallantoic membrane revealed the anastomosis of vessels in the chorioallantoic membrane with newly formed sinusoids in the reconstructing liver (Fig. 4g). By eleven days post-transplantation, structures similar to the bile duct (Fig. 4d arrowhead), central vein (Fig. 4d arrow) and hepatic cords (Fig. 4d) appeared in the reconstructing liver. Immunohistochemistry for albumin also revealed that the hepatocytes in this period produced albumin (Fig. 4f). Serial semi-thin sections showed that clusters of blood cells in the regenerated region were not in the sinusoid but confined to a space surrounded directly by hepatocytes (Fig. 5a-h arrowheads).

With transplantation of the dissociated liver cells, cells that were immersed in the chorioallantoic membrane proliferated and formed small clusters (Fig. 6a). Two types of cell clusters were then observed in the chorioallantoic membrane: one consisted of only hepatocytes, where neither hematopoiesis nor sinusoids were observed (Fig. 6b), the other was of hepatocytes with hematopoiesis and sinusoids (Fig. 6c).

\section{Discussion}

The present study investigated the process of liver regeneration as performed in the grafted liver tissue or dissociated liver cells of the 6-day-old chick embryo onto the chorioallantoic membrane of a 9-day-old chick 
embryo. Transplantation of the liver tissue showed that cells in the large central part of the graft died soon after transplantation, while cells in the superficial zoneeither exposed to the air or adhering to the chorioallantoic membrane-survived, though the former zone eventually disappeared. This zone was, however, thicker than the zone adhering to the chorioallantoic membrane at one day post-transplantation. This finding indicated that the cells in the superficial zone exposed to the air were able to obtain sufficient oxygen directly from the air for their survival (Awwad et al., 1986) at an early date. However, this zone could not receive the blood circulation, and the reception of nutrients in this zone became very limited due to its isolation from vessels of the chorioallantoic membrane. As a result, the superficial zone exposed to the air soon disappeared because the hepatocytes exhausted their intrinsic nutrients. On the other hand, cells in the zone adhering to the chorioallantoic membrane were fewer than those in the zone exposed to the air in the early stage, probably because the former could not obtain as much oxygen as the latter. However, the cells in this zone eventually proliferated and reconstructed the liver, a reasonable outcome if we noticed the appearance of anastomosis between vessels of chorioallantoic membrane and newly formed sinusoids in the reconstructing liver afterward. The cells in this zone could obtain nutrition and oxygen directly and continuously from blood of the chorioallantoic membrane. These findings also accord with those by Ausprunk et al. (1975), who stated that the continuity of newly formed vessels in the graft with host vessels was necessary for the successful transplantation of the graft on the chorioallantoic membrane.

Our transplantation studies using dissociated cells of the whole liver grafted onto the chorioallantoic membrane showed that hematopoiesis appeared in the reconstructed liver in the same way as in the case of transplantation of liver pieces. It is conceivable that hepatocytes and/ or hepatic stem cells and hematopoietic stem cells are included in the dissociated cells of whole liver. When these cells are transplanted onto the chorioallantoic membrane, three types of cell combinations can be created: hepatocytes and/or hepatic stem cells alone, hematopoietic stem cells alone, and hepatocytes and/ or hepatic stem cells with hematopoietic stem cells. Among these, the mass of hematopoietic stem cells alone will construct blood cells and vessels, but they cannot be distinguished from blood cells and vessels of the host in this experimental design. However, clusters composed of hepatocytes alone or those of hepatocytes with hematopoiesis were clearly observed in the present study. One noteworthy finding was that clusters formed with hepatocytes alone were small, while clusters of hepatocytes mixed with numerous blood cells were relatively large in size. This finding indicates that the combination of hepatocytes and/or hepatic stem cells with hematopoietic stem cells is important for liver reconstruction. A similar finding was made by Ohashi $e t$ al. (2007), who produced hepatic cell sheets composed only of hepatocytes in culture, transplanted these sheets into the subcutaneous tissues, and maintained them for 200 days. During this period, hepatocytes proliferated and produced a thick mass, but sinusoids did not appear among them. Thus, we consider that the proliferation of hepatocytes with accompanying hematopoiesis by hematopoietic stem cells is important for reconstructing the liver tissue with sinusoids.

When hematopoiesis occurs in transplants, proliferating blood cells may push away the cluster of hepatocytes, resulting in the formation of spaces for blood cells directly surrounded by hepatocytes. Our semi-thin section studies showed that there were no endothelial cells lining any of these spaces, indicating that the spaces were produced and expanded by filling up a number of blood cells. Thus, it is probable that such a space is connected with a neighboring newly formed space and/or already formed sinusoids, and finally become sinusoids. In other words, hematopoiesis may be essential for the formation of sinusoidal spaces in the regenerated liver. However, in the present study, we could not clarify how the endothelial cell lined the sinusoid spaces, nor from where those endothelial cells were derived. Further studies are needed to clarify this point.

It is difficult to determine whether the regenerated liver in this research acquired the normal structure because the chicken liver had no clear hepatic lobules. However, we showed that the reconstructing liver had structures similar to hepatic cords, sinusoids, a central vein, and bile ducts, and that the hepatocytes produced albumin. These indicate that the chorioallantoic membrane can provide environment for liver regeneration. Our findings suggest that the liver does not regenerate normally by transplantation of hepatocytes without accompanying hematopoietic tissues because sinusoids can not be constructed. We therefore consider that hematopoiesis may be a key factor for the formation of sinusoids, resulting in the reconstruction of the liver with both normal structure and function.

\section{Acknowledgements}

We thank Ms. Chihiro Ohta for her technical assistance. 


\section{References}

Ausprunk DH, Knighton DR, Folkman J: Vascularization of normal and neoplastic tissues grafted to the chick chorioallantois. Role of host and preexisting graft blood vessels. Am J Pathol 79: 597-628 (1975).

Awwad HK, el Naggar M, Mocktar N, Barsoum M: Intercapillary distance measurement as an indicator of hypoxia in carcinoma of the cervix uteri. Int J Radiat Oncol Biol Phys 12: 1329-1333 (1986).

Bertossi M, Virgintino D, Coltey P, Errede M, Mancini L, Roncali L: Vascularization of embryonic adrenal gland grafted onto chorioallantoic membrane. Anat Embryol (Berl) 198: 267-275 (1998).

Feng ZQ, Chu X, Huang NP, Wang T, Wang Y, Shi X, Ding Y, Gu ZZ: The effect of nanofibrous galactosylated chitosan scaffolds on the formation of rat primary hepatocyte aggregates and the maintenance of liver function. Biomaterials 30: 2753-2763 (2009).

Hamburger V, Hamilton $\mathrm{H}$ : A serial of normal stages in the development of the chick embryo. J Morphol 88: 49-92 (1951).

Hancox NM: The survival of transplanted embryo bone grafted to chorioallantoic membrane, and subsequent osteogenesis. J Physiol 106: 279-285. (1947).

Harada K, Mitaka T, Miyamoto S, Sugimoto S, Ikeda S, Takeda H, Mochizuki Y, Hirata K: Rapid formation of hepatic organoid in collagen sponge by rat small hepatocytes and hepatic nonparenchymal cells. $J$ Hepatol 39: 716-723 (2003).

Katoh M, Nakada K, Miyazaki JI: Liver regeneration on chicken chorioallantoic membrane. Cell Tiss Org 169: 125-133 (2001).

Michalopoulos GK, DeFrances MC: Liver regeneration. Science 276: 60-66 (1997).

Mitaka T, Kojima T, Mizuguchi T, Mochizuki Y: Growth and maturation of small hepatocytes dissociated from adult rat liver. Biochem Biophys Res Commun 214: 310-317 (1995).
Mito M, Ebata H, Kusano M, Onishi T, Saito T, Sakamoto S: Morphology and function of dissociated hepatocytes transplanted into rat spleen. Transplant 28: 499-505 (1979).

Murphy JB: The effect of adult chicken organ grafts on the chick embryo. J Exp Med 24: 1-5 (1916).

Nakada K, Yao Y, Mashima J, Katoh M, Miyazaki J, Hirabayashi T: Skeletal muscle regeneration induced by chorio-allantoic grafting. J Muscle Res Cell Motil 19: 169-177 (1998).

Nijweide PJ, van Iperen-van Gent AS, Kawilarang-de Haas EW, van der Plas A, Wassenaar AM: Bone formation and calcification by dissociated osteoblast like cells. J Cell Biol 93: 318-323 (1982).

Ohashi K, Yokoyama T, Yamato M, Kuge H, Kanehiro H, Tsutsumi M, Amanuma T, Iwata H, Yang J, Okano T, Nakajima Y: Engineering functional two- and threedimensional liver systems in vivo using hepatic tissue sheets. Nat Med 13: 880-885 (2007).

Sezer N: Cultivation of conjunctival and corneal tissue on the chorioallantoic membrane. AMA Arch Ophthalmol 44: 703-709. (1950).

Shibata C, Mizuguchi T, Kikkawa Y, Nobuoka T, Oshima H, Kawasaki H, Kawamoto M, Katsuramaki T, Mitaka $\mathrm{T}$, Hirata K: Liver repopulation and long-term function of rat small hepatocyte transplantation as an alternative cell source for hepatocyte transplantation. Liver Transpl 12: 78-87. (2006).

Sudo R, Mitaka T, Ikeda M, Tanishita K: Reconstruction of 3D stacked-up structures by rat small hepatocytes on microporous membranes. FASEB J 19: 1695-1697 (2005).

Wen F, Chang S, Toh YC, Arooz T, Zhuo L, Teoh SH, Yu H: Development of dual-compartment perfusion bioreactor for serial coculture of hepatocytes and stellate cells in poly (lactic-co-glycolic acid)-collagen scaffolds. J Biomed Mater Res B Appl Biomater 87: 154-162 (2008). 